\title{
Comparative analysis of target gene exon sequencing by cognitive technology using a next generation sequencing platform in patients with lung cancer
}

\author{
YU CHEN $^{1,2}$, WENQING YAN ${ }^{1,2}$, ZHI XIE ${ }^{1,2}$, WEIBANG GUO $^{1,2}$, \\ DANXIA LU ${ }^{1,2}$, ZHIYI LV ${ }^{1,2}$ and XUCHAO ZHANG ${ }^{1,2}$ \\ ${ }^{1}$ Guangdong Provincial Key Laboratory of Translational Medicine in Lung Cancer, Medical Research Center, \\ Guangdong Provincial People's Hospital, Guangdong Academy of Medical Sciences, School of Medicine, \\ South China University of Technology, Guangzhou, Guangdong 510080; \\ ${ }^{2}$ Guangdong Provincial Key Laboratory of Translational Medicine in Lung Cancer, Medical Research Center, \\ Guangdong Provincial People's Hospital, Guangdong Academy of Medical Sciences,
}

The Second School of Clinical Medicine, Southern Medical University, Guangzhou, Guangdong 510280, P.R. China

Received March 31, 2020; Accepted December 9, 2020

DOI: $10.3892 / \mathrm{mco} .2020 .2198$

\begin{abstract}
Next generation sequencing (NGS) technology is an increasingly important clinical tool for therapeutic decision-making. However, interpretation of NGS data presents challenges at the point of care, due to limitations in understanding the clinical importance of gene variants and efficiently translating results into actionable information for the clinician. The present study compared two approaches for annotating and reporting actionable genes and gene mutations from tumor samples: The traditional approach of manual curation, annotation and reporting using an experienced molecular tumor bioinformationist; and a cloud-based cognitive technology, with the goal to detect gene mutations of potential significance in Chinese patients with lung cancer. Data from 285 gene-targeted exon sequencing previously conducted on 115 patient tissue samples between 2014 and 2016 and subsequently manually annotated and evaluated by the Guangdong Lung Cancer Institute (GLCI) research team were analyzed by the Watson for Genomics (WfG) cognitive genomics technology. A comparative analysis of the annotation results of the two methods was conducted to identify quantitative and qualitative differences in the mutations generated. The complete congruence rate of annotation results between WfG
\end{abstract}

Correspondence to: Professor Xuchao Zhang, Guangdong Provincial Key Laboratory of Translational Medicine in Lung Cancer, Medical Research Center, Guangdong Provincial People's Hospital, Guangdong Academy of Medical Sciences, School of Medicine, South China University of Technology, 106 Zhongshan Er Road, Guangzhou, Guangdong 510080, P.R. China

E-mail: zhxuchao3000@126.com

Key words: next generation sequencing, lung cancer, precision medicine, artificial intelligence, genomics analysis and the GLCI bioinformatician was $43.48 \%$. In 65 (56.52\%) samples, WfG analysis identified and interpreted, on average, 1.54 more mutation sites in each sample than the manual GLCI review. These mutation sites were located on 27 genes, including EP300, ARIDIA, STK11 and DNMT3A. Mutations in the EP300 gene were most prevalent, and present in $30.77 \%$ samples. The Tumor Mutation Burden (TMB) interpreted by WfG analysis (1.82) was significantly higher than the TMB (0.73) interpreted by GLCI review. Compared with manual curation by a bioinformatician, WfG analysis provided comprehensive insights and additional genetic alterations to inform clinical therapeutic strategies for patients with lung cancer. These findings suggest the valuable role of cognitive computing to increase efficiency in the comprehensive detection and interpretation of genetic alterations which may inform opportunities for targeted cancer therapies.

\section{Introduction}

Lung cancer, common worldwide, is the leading cause of cancer deaths in China. Effective and efficient measures to target therapies are needed to reduce disease burden and improve patient prognoses and outcomes. Precision medicine uses genomic analysis, including next generation sequencing, to identify the genetic profile of individual patients and lung cancer cells to identify specific individual receptivity to available therapies. The 2015 launch of the Precision Medicine Initiative by US President Obama accelerated the integration of next generation sequencing (NGS) methods in genomic medicine, especially in oncology care $(1,2)$. NGS technology is becoming more widely employed as a novel genetic screening, prognostic and diagnostic technique for clinical disease management (3-5) and is becoming an effective and acceptable method for clinical gene detection $(6,7)$. Although it is still in the early stage of clinical application for the diagnosis and treatment of tumors (8-10), its continuous innovation has generated increased awareness and interest in the role of 
genetic markers and the molecular mechanisms of diseases. Genome alterations play a significant role in disease recurrence for lung cancer (11). Large-scale genomic sequencing studies have revealed the complex genomic landscape of lung cancer, with tumor heterogeneity (12).

NGS shows promise in the treatment of lung cancer to identify candidate biomarkers for early diagnosis, identify prognostic factors, and detect actionable mutations to guide targeted therapy decisions (13). However, NGS generates massive volumes of data. Analyzing and interpreting this data creates challenges for analysts and clinicians alike. As a result, the analysis of data and annotation of variation have become a major bottleneck that inhibits wider clinical adoption and usefulness of NGS technology.

There are many tools available to analyze NGS data for variants (14). The standard method for annotation of NGS mutations at cancer care centers like Guangdong Lung Cancer Institute (GLCI) includes retrieval, analysis and comparison of the annotation results with databases like DrugBank, COSMIC, dbSNP, OMIM, ClinVar, 5000 Exomes and 1000 Genomes. Genes are further analyzed through screening under a series of conditions examining zygosity, variant type, variant effect, location, filtered coverage and minor allele frequency, on the basis of preliminary analysis of NGS data.

This study compared the annotation and interpretation of NGS results at a large volume cancer center in Guangdong, China using standard methods and using IBM Watson for Genomics (WfG), a cloud-based cognitive computing system. WfG is trained to analyze molecular data at a massive scale to provide clinically actionable insights that are supported by all available relevant evidence. The tool is built on several different predictive models that can perform analysis across the whole-genome and accesses a comprehensive database of structured and unstructured data sources using Natural Language Processing (NLP). (Over 200 sources include DrugBank, NCI, COSMIC, ClinVar, and 1000 Genomes, as well as evidence extracted from the universe of biological and medical literature.) WfG is in use in selected markets $(13,15,16)$, however, its application in the analysis and annotation of results of NGS data in Chinese patients has not been reported. This study examined and compared the NGS data annotation process by comparing the results of the gene mutation annotation for Chinese patients with lung cancer generated by Guangdong Lung Cancer Institute (GLCI) bioinformaticians and WfG. The ultimate goal of this study was to leverage insights from the analysis to inform individual treatment decisions to benefit future Chinese patients with lung cancer.

\section{Materials and methods}

Materials. In terms of patient specimen collection and analysis, we do have approval from our institutional ethics board and informed consent from each patient. Actually, we have established a tissue repository center (Tumor Sample Bank) in our cancer center which was approved by Human Biomaterial and Genetic Resource Office of China. Researchers at Guangdong Lung Cancer Institute (GLCI) obtained a variety of tissue samples from the Tumor Sample Bank in GLCI of Guangdong Provincial People's Hospital, Guangzhou, China. The samples, from 115 randomly selected patients diagnosed with lung cancer at Guangdong General Hospital between 2014 and 2016, included 10 formalin fixed paraffin-embedded (FFPE) samples, 12 small samples collected after puncture, 4 plasma samples and 89 large tumor samples collected during surgery. All patients were well informed and signed the informed consent.

DNA extraction. Researchers used QIAGEN Q1AampDNA Mini Kit and QIAGEN QlAampBlood Mini Kit for the gDNA extraction process from each tissue sample (i.e., the FFPE samples, small samples collected after puncture and large samples collected in surgery) and DNA from 1-4 ml plasma samples, respectively. DNA quantitative analysis was completed using Qubit analyzer.

Design and synthesis of target capture probe. Hybrid capture in target areas was implemented using SureSelect ${ }^{\mathrm{XT}}$ Custom library. This probe library was designed through SureDesign software based on genome hg19/GRCh37; target areas were lung cancer-related high-frequency mutation gene exon areas.

Establishment of NGS library. The NGS library was established using free DNA in plasma and gDNA in tissue samples. Free DNA was extracted from 1-2 ml plasma to establish the library, without requirement of fragmentation. Approximately, 50-1,000 ng of gDNA was extracted from each tissue sample and cut into 100-200 bp segments through enzyme digestion to establish the library. The NGS library was established using Ion Xpress ${ }^{\mathrm{TM}}$ Plus Fragment Library Kit and Ion Xpress ${ }^{\mathrm{TM}}$ Barcode Adapters 1-16 Kit, and the selection, purification and recovery of DNA was completed via Agencourt AMPure XP beads. Target areas were captured after hybridization through SureSelectXT Custom library at $65^{\circ} \mathrm{C}$ for $16-24 \mathrm{~h}$ following 11 cycles of polymerase chain reaction (PCR) amplification in pre-library after segment selection. The target area sequencing library was obtained from the captured library after purification and 9 cycles of PCR amplification. Finally, QIAxcel and Qubit were used to detect the library segment length and library concentration, respectively.

Sequencing and data analysis. The library was diluted into $12 \mathrm{pM}$ using water according to its concentration and connected with microballoon using Ion PI ${ }^{\mathrm{TM}}$ Template OT2 200 Kit v2, after which the samples were spotted on P1 chips for sequencing. The sequencing data were compared with human genome $\mathrm{Hg} 19$ using Suite software (Life Technologies, Version 5.0.2), and mutations were detected using Variant Caller software (Life Technologies, Version 5.0.2.1), so as to form corresponding variant calling files (VCF).

Target sequencing data interpretation by GLCI using standard methods. Integrative Genomics Viewer (IGV) software was applied by GLCI bioinformaticians to annotate the gene mutation information by comparing with the databases DrugBank, COSMIC, dbSNP, OMIM, ClinVar, 5000 Exomes and 1000 Genomes.

Gene variant interpretation executed by Watson for Genomics. Upon sequence completion by GLCI lab, the 


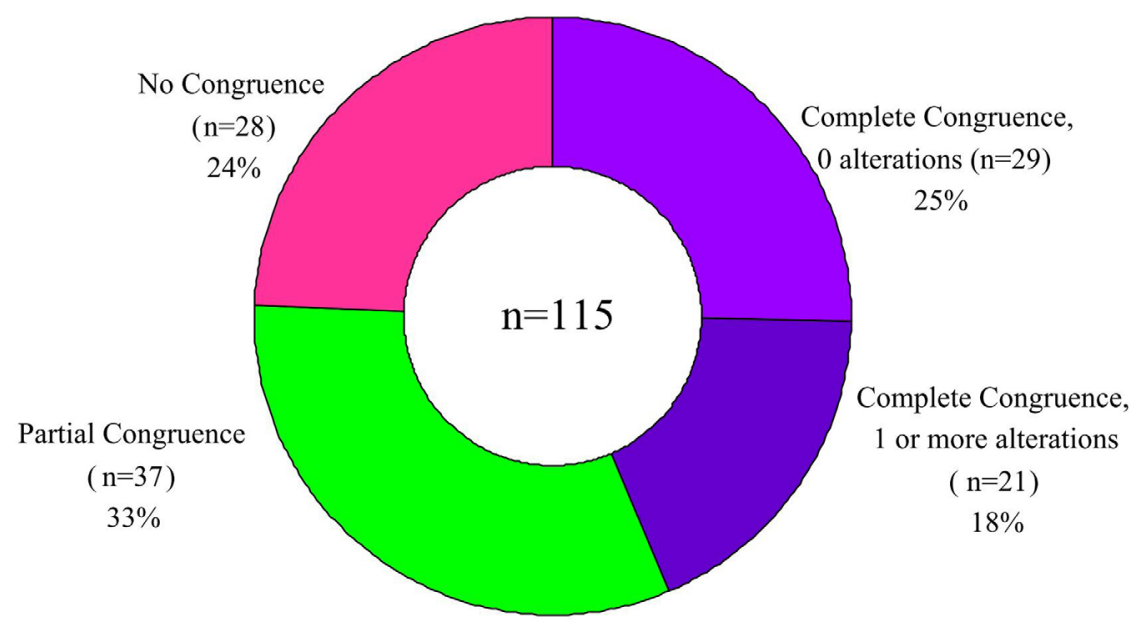

Figure 1. Comparing patient-paired samples using different annotation methods of Watson for Genomics and Guangdong Lung Cancer Institute bioinformationist.

research team accessed the cloud-based cognitive computing tool, Watson for Genomics (WfG). The following information was uploaded to WfG: (a) tumor type, (b) a list of variants as a variant calling file (.vcf). Uploading this data required approximately $1 \mathrm{~min}$ for each sample, by a data technician. After these data were uploaded, WfG performed the Molecular Profile Analysis (MPA) for each gene with a variant. A subpart of the WfG cognitive tool, the MPA reviews evidence from functional studies and protein structure and applies programming logic to classify variants into five categories: Pathogenic, likely pathogenic, benign, likely benign, and variables of unknown significance (VUS). Alterations categorized as benign or likely benign were removed from the report. Next, WfG identified a gene as actionable if: a) the variant was pathogenic or likely pathogenic; b) the variant was directly targetable or part of a pathway that was targetable based on evidence from the literature; and c) a U.S. Food and Drug Administration-approved or investigational target therapy was available.

Statistical analysis. Statistical analysis of the difference between tumor mutation burden obtained by WFG and GLCI was performed by the paired t-test analysis. Counting data was expressed by the number of cases/percentage $n(\%)$, and the measurement data were expressed by mean number (mean \pm SD). t-test analysis was performed using SPSS v22.0 software (IBM Corp.). $\mathrm{P}<0.05$ was considered to indicate a statistically significant difference.

\section{Results}

Congruence rate of gene mutation interpretation results of GLCI and WfG analyses/between two methods. 'Complete congruence' was defined as having completely consistent interpretation results from the two methods, which included the following two scenarios: In the first scenario, no mutation sites were identified by either method, i.e., Complete Congruence, 0 alterations reported. In the second scenario, the same single or multiple mutation sites were interpreted by both methods, i.e., Complete Congruence, 1 or more identical alterations reported by both methods.
'Partial congruence' was defined as the partially consistent interpretation results from the two methods, i.e., mutation sites interpreted by WfG analysis contained those by GLCI bioinformaticians. 'No congruence' was defined as totally inconsistent interpretation results from the two methods, i.e., no mutation sites were interpreted by GLCI bioinformaticians while 1 or more mutation site were interpreted by WfG analysis, or vice versa.

After annotation of the sequencing results of all 115 samples, across all samples WfG identified 180 alterations whereas GLCI identified 80 mutation sites. The congruence rate in detecting mutation sites across the entire sample was $44.44 \%$. Complete congruence was found in the analyses of 50 samples (congruence rate $43.48 \%$.) Of these completely congruent samples, 29 had no reported mutation sites (Complete Congruence, 0 alterations), and 21 had 29 mutation sites (Complete Congruence, 1 or more alterations).

In the remaining 65 samples $(56.52 \%)$, a total of 180 mutation sites were found after analysis by both methods. Compared with GLCI bioinformaticians, WfG analysis interpreted more mutation sites (100), with an average of 1.54 more mutation sites in each sample. (In one sample, WfG interpreted 11 more mutation sites than were interpreted by the GLCI bioinformatician). In 37 samples (32.17\%) Partial Congruence was found in the analysis: GLCI interpreted 51 mutation sites and WfG interpreted 103 mutation sites. (WfG interpreted an average of 1.41 more mutation sites in each sample). Finally, in the 28 samples with no congruence, WfG reported 48 mutation sites and GLCI reported zero mutation sites. In these samples, WfG analysis interpreted 1.71 more mutation sites in each sample when compared to GLCI analysis WfG (Fig. 1 and Table I).

Differential genes and signal pathways in data interpretation results between two methods. NGS data from 115 samples were annotated by WfG and GLCI bioinformaticians respectively. In 50 samples (43.5\%), no mutations were found by either GLCI or WfG. However, in the remaining 65 samples (56.5\%), WfG analysis interpreted more mutation sites (100), whereas in $54(47.0 \%)$ samples, WfG analysis interpreted 1-2 more mutation sites when compared with GLCI bioinformaticians' results (Table II). 
Table I. Number of mutation sites identified by WfG and GLCI.

\begin{tabular}{lccc}
\hline Type of uniformity & No. & $\begin{array}{c}\text { Mutation sites } \\
\text { identified by WfG, n }\end{array}$ & $\begin{array}{c}\text { Mutation sites } \\
\text { identified by GLCI, } \mathrm{n}\end{array}$ \\
\hline Complete congruence, 0 alterations & 29 & 0 & 0 \\
Complete congruence, 1 or more alterations & 21 & 29 & 29 \\
Partial congruence & 37 & 103 & 51 \\
No congruence & 28 & 48 & 0 \\
Total & 115 & 180 & 80 \\
\hline
\end{tabular}

GLCI, Guangdong Lung Cancer Institute; WfG, Watson for Genomics.

Table II. Variability in mutation sites interpreted by Watson for Genomics and Guangdong Lung Cancer Institute.

\begin{tabular}{lccc}
\hline $\begin{array}{l}\text { Variability (number of } \\
\text { different mutation sites) }\end{array}$ & $\begin{array}{c}\text { Incidence, } \\
\text { no. of samples }\end{array}$ & $\begin{array}{c}\text { Share of } \\
\text { samples, } \%\end{array}$ & $\begin{array}{c}\text { Cumulative } \\
\text { percentage, } \%\end{array}$ \\
\hline 0 & 50 & 43.5 & 43.5 \\
1 & 41 & 35.7 & 79.1 \\
2 & 13 & 11.3 & 90.4 \\
3 & 3 & 2.6 & 93.0 \\
4 & 4 & 3.5 & 96.5 \\
5 & 2 & 1.7 & 98.3 \\
6 & 1 & 0.9 & 99.1 \\
11 & 1 & 0.9 & 100.0 \\
Total & 115 & 100.0 & \\
\hline
\end{tabular}

The 100 mutation sites that were additionally identified by WfG analysis were located on 27 genes, including EP300, ARIDIA, STK11 and DNMT3A. The mutation rate was $30.77 \%$ $(20 / 65)$ in EP300 gene; $18.46 \%(12 / 65), 16.92 \%(11 / 65)$ and $13.85 \%(9 / 65)$ in ARIDIA, STK 11 and DNMT3A genes, respectively. In the $37(32.17 \%)$ samples out of 65,51 mutation sites were interpreted by GLCI bioinformaticians while 103 by WfG analysis, with 51 co-interpreted mutation sites located on EGFR, KRAS and TP53 genes. WfG interpreted 52 more mutation sites located on EP300, ARID1A, STK11, DNMT3A, PIK3R1, PTEN, ATM, BCL2, MSH6, NF1, PTPN11, ATR, $B R C A 1, C D K N 2 A, C R E B B P, M E T, R N F 43$ and TSC1 genes. In another $28(24.35 \%)$ samples out of 65 , no mutation sites were interpreted by GLCI bioinformaticians while 48 mutation sites were interpreted by WfG analysis and were located on EP300, ARID1A, STK11, TSC2, BRCA2, MSH6, BRCA1, DNMT3A, NF2, ATM, PIK3R1, PTEN, BRAF, CDKN1B, IDH1, IDH2, KIT, PMS2, RNF43 and TSC1 genes (Fig. 2).

Analysis of congruence rate of common driver gene mutation annotation. The two methods reported similar annotation results in the mutations analysis of 4 common driver genes of EGFR, KRAS, TP53 and ERBB2. Of the 115 samples, the total number of mutation sites for these 4 genes was 82,80 of which were identically reported by both methods (congruence rate $97.56 \%$ ). The congruence rates of EGFR, KRAS, TP53 and ERBB2 were $100 \%$ (29/29), 100\% (12/12), 94.87\% (37/39) and $100 \%(2 / 2)$ respectively (Fig. 3).
Analysis of tumor mutation burden (TMB) interpretation results between two methods. The average number of missense mutations obtained before preliminary analysis and filtration of NGS data of target $0.947 \mathrm{~kb}$ genes in the 115 samples was 130.43, with a TMB of 137.78. After WfG interpretation and filtration, the average number of missense mutations and TMB obtained was 1.72 and 1.82, significantly higher than the 0.69 and 0.73 , respectively, obtained by GLCI bioinformaticians $(\mathrm{P}<0.05)$ (Fig. 4).

New high-Minor Allele Frequency (MAF) mutations indicated by WfG. In one of the 37 samples with Partial Congruence, (sample no. 29002) WfG analysis identified 4 new mutation sites with high MAF. The MAF of TP53 G245V mutation found by both methods was $5.25 \%$, while the MAF was $11.95,14.05,35.15$ and $32.02 \%$ in new mutation sites $A T R$ G492fs, DNMT3A A222fs, PTEN R130Q and STK11 G257fs interpreted by WfG only, respectively (Fig. 5).

\section{Discussion}

Several cancer centers are beginning to use artificial intelligence (AI) computing systems to analyze NGS data (14). The speed and volume of research, discovery and reporting on new genes and mutations, and their relationships to tumorigenesis is accelerating. Monitoring and integrating this knowledge for use in clinical decision-making is a task well-suited to cognitive computing technologies. WfG is a cognitive computing 
A

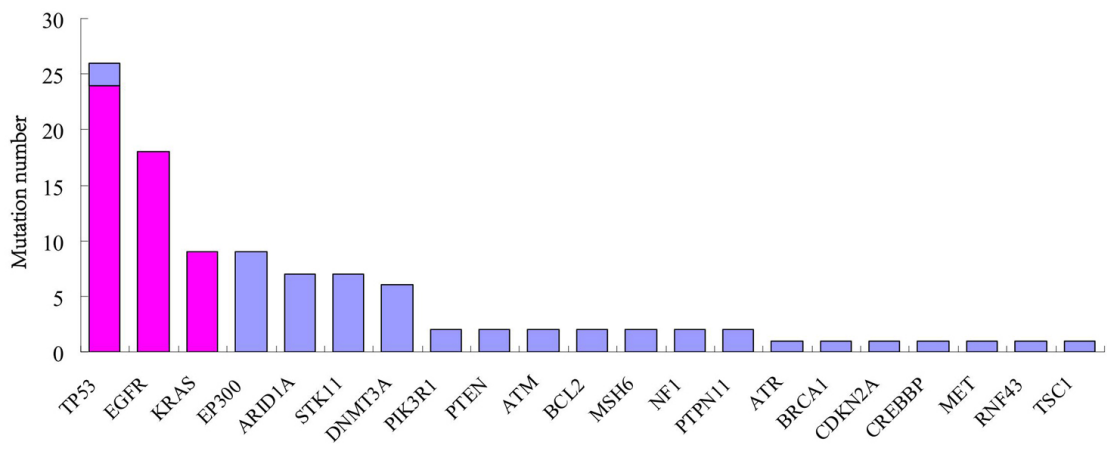

B

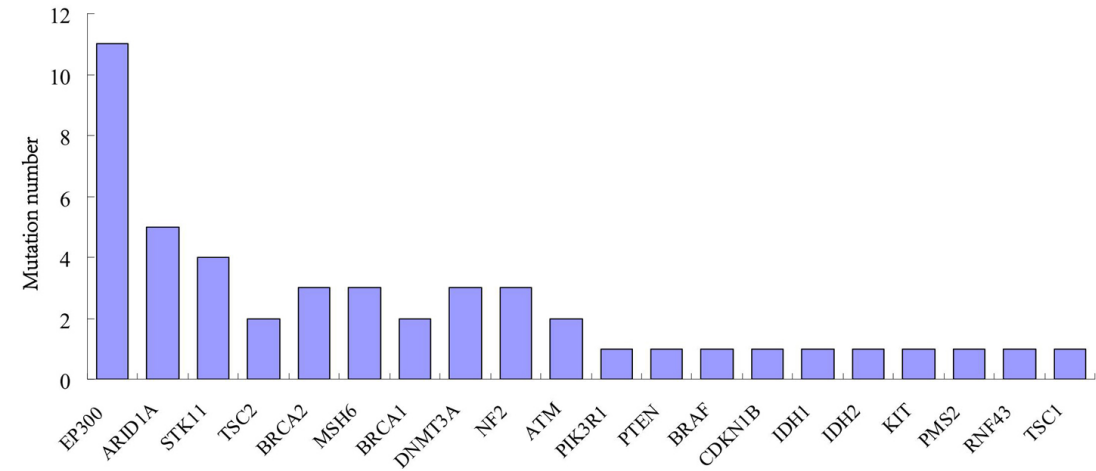

Figure 2. Mutation genes identified by GLCI and WfG. (A) Partial congruence and (B) no congruence. GLCI, Guangdong Lung Cancer Institute; WfG, Watson for Genomics.

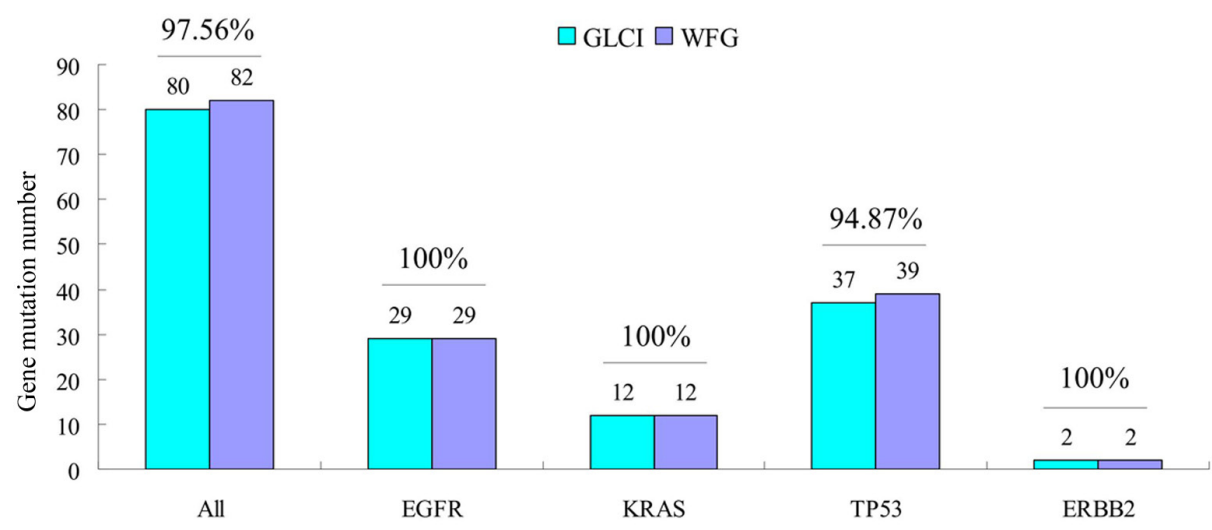

Figure 3. Congruence rates of common driver gene mutation in analysis by both methods. GLCI, Guangdong Lung Cancer Institute; ERBB2, erb-b2 receptor tyrosine kinase 2; WfG, Watson for Genomics.

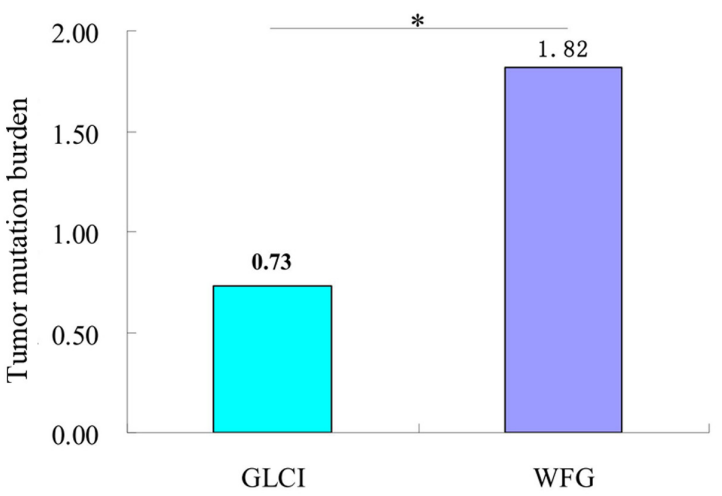

Figure 4. Comparison of tumor mutation burden (across all samples) for the two methods. ${ }^{*} \mathrm{P}<0.05$. GLCI, Guangdong Lung Cancer Institute; WfG, Watson for Genomics. technology which can learn new information and analyze data at a rate that far exceeds manual curation and analysis (14). During this study, the number of cases analyzed and annotated by one GLCI bioinformatician working full time was approximately 10 cases per one week. In contrast, WfG completed analysis and annotation of each sample in $\sim 3$ min, evidence that WfG was able to perform this analysis at a much faster rate than even a highly trained human analyst.

To compare the effectiveness of the WfG cognitive computing tool with human-only targeted panels at identifying potentially actionable gene mutations for Chinese patients with lung cancer, we retrospectively analyzed 115 cases from GLCI that had undergone targeted DNA sequencing of 285 genes and subsequent analysis by the GLCI bioinformaticians. We conducted an independent analysis of these 115 samples using WfG. WfG 


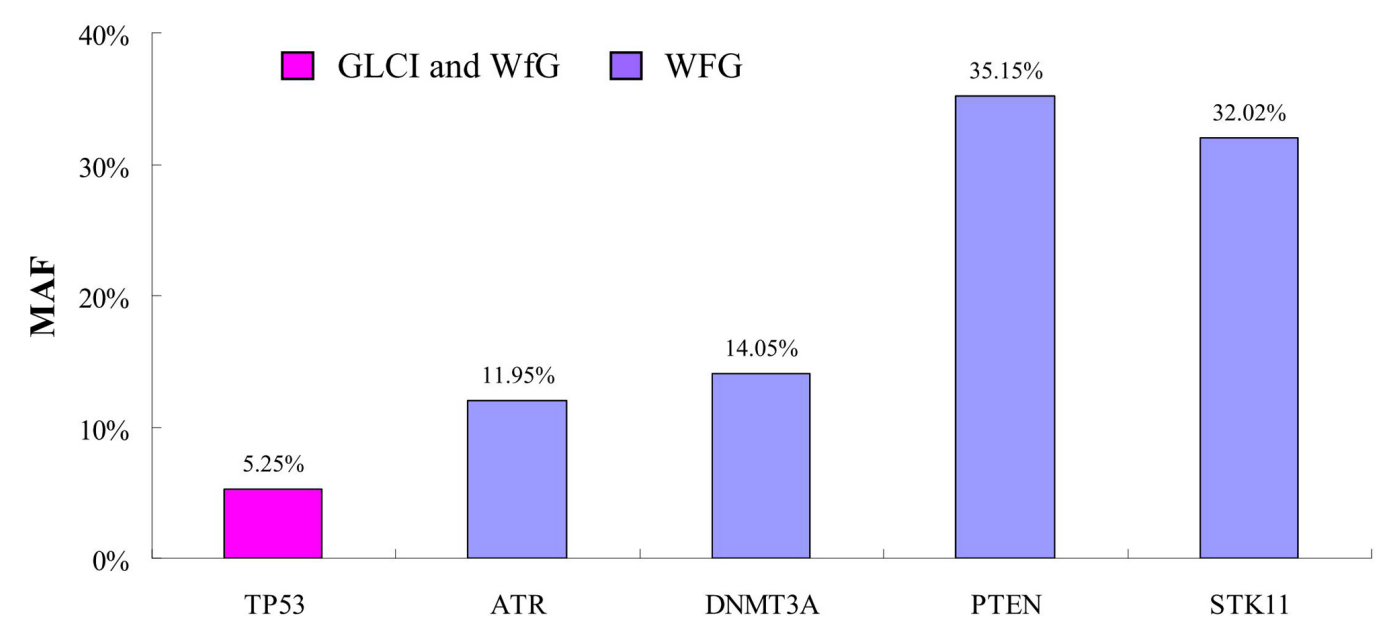

Figure 5. MAF of five mutation sites in one sample (no. 29002) as interpreted by both methods. MAF, minor allele frequency; ATR, ATR serine/threonine kinase; DNMT3A, DNA methyltransferase 3 $\alpha$; STK11, serine/threonine kinase 11; GLCI, Guangdong Lung Cancer Institute; WfG, Watson for Genomics.

was provided the full list of variants on each sequenced sample and identified 180 mutation sites in the 115 samples vs. only 80 mutation sites identified by GLCI bioinformaticians. This indicated that WfG could interpret more mutation sites than GLCI bioinformaticians using standard methods. This is particularly valuable as it is well known that target therapy or individualized therapy focuses on specific variant genes or specified gene mutation sites. WfG's ability to interpret more gene mutation sites from $56.52 \%$ samples demonstrates that it can provide more opportunities for targeted therapy for patients with lung cancer and additional useful information for clinical doctors developing therapeutic strategies.

In this study, the congruence rate of the analyses of NGS results was $43.48 \%$ and congruence rate of mutations was 44.44\% between WfG analysis and GLCI bioinformaticians', and is similar to the results of other studies which have compared WfG and manual mutation analysis $(13,16)$. There are several possible reasons that fewer mutation sites were interpreted by GLCI bioinformaticians than by WfG. For example, the GLCI biological analysis was completed by one individual bioinformatician and was relatively conservative, emphasizing mutation sites that were common driver gene mutation sites that had been studied thoroughly, as demonstrated by the $97.56 \%$ congruence rate of significant driver gene mutation sites. This is because GLCI bioinformaticians mainly focus on driving genes, while the new analysis methods are more comprehensive, which not only does not lose the driving gene targets, but also increases the discovery of many new rare mutation targets. A defining feature of the WfG cognitive tool and its analysis is its ability to retrieve almost all the research published worldwide, and to extract actionable information to analyze and annotate gene mutation sites. In contrast, the bioinformatician relied on extensive individual experience in reading the literature and analyzing results. As to most uncommon variant genes, WfG annotation was superior to the GLCI bioinformatician's. In this study results, the genes in the list interpreted by WfG analysis only, such as MSH6, DNMT3A, NF2, ATM, PIK3R1, CDKN1B, IDH1, $I D H 2, K I T, P M S 2, R N F 43$ and TSC1, reflect WfG's ability to access and integrate more recent research consistently during analysis. This may also explain discrepancies between the annotation results of bioinformaticians and WfG analysis in samples with mutation sites occurring in uncommon genes. This may also suggest that uncommon variant genes should be of increased concern to bioinformaticians, especially in this rapidly evolving arena.

This retrospective analysis of individual tissue samples representing 115 cases offers an opportunity to review treatment therapies with the benefit of new information gleaned from WfG. In $24.35 \%$ samples, targeted therapy was not performed for patients as no clinically significant mutation sites were found by GLCI bioinformaticians. In contrast, mutation sites were found in uncommon genes by WfG analysis in $56.52 \%$ of the samples. Patients with these mutations may have been eligible to participate in clinical trials with corresponding agents, had this information been available to their clinicians at the point of care. Going forward, it is therefore possible that cognitive technologies such as WfG will be able to assist clinicians by providing the comprehensive and timely analysis needed to help them guide patients to appropriate therapies. This is consistent with the view of Itahashi et al (17). They believe that WFG is useful for a clinician at a general hospital additional survey of evidence by a clinician is required when evaluating functions (17). In our sample of Chinese patients with lung cancer, opportunities for targeted therapy might have been available for $24.35 \%$ of patients, informed by the WfG analysis. Of particular interest, in one sample in which there was no congruence between GLCI and WfG analysis, and in which there was no common mutation gene variation, this analysis found that the MAF value of multiple new gene mutation sites interpreted by WfG analysis was evidently higher than that of discovered insignificant gene mutation sites. This finding suggests that the therapeutic strategies for patients with findings like these might be changed accordingly.

This report is a preliminary study to compare AI-aided analysis using the cognitive computing technology WfG to the standard manual method. Limitations of the study included relatively small sample size, absence of in-association analysis with clinical therapy and, as a result, no specific information about how the results of this analysis might have affected clinical outcomes. Our study did not systematically measure 
the time required to annotate, interpret and report NGS results by either the standard method used by GLCI, or by WfG. Future studies will be necessary to address these limitations.

In conclusion, this study provides evidence that analysis of NGS results by the cognitive computing technology WfG can provide an accurate and comprehensive interpretation of more gene mutation sites through a more rapid process than routine manual analysis, generating potential opportunities of targeted therapy for cancer patients. The basis of targeted therapy is to obtain the effective gene mutation information of patients in time. For patients diagnosed with lung cancer in China and elsewhere, the timely provision of actionable information that may affect treatment options can be critical to individual therapy and research. The abundance of mutation sites interpreted uniquely by WfG analysis in partial samples was relatively high, further suggesting opportunities to optimize clinical decision making for a greater number of affected patients in the future.

\section{Acknowledgements}

This abstract was presented at the 2018 ASCO Annual Meeting (June 1, 2018; Chicago, USA) and was published as Abstract no. e24254.

\section{Funding}

The present study was supported by the following grants: Guangdong Provincial Natural Science Program (grant no. 2019A1515010900; to XZ); GDPH Dengfeng Program (grant nos. DFJH201903, KJ012019444 and 8197103306; to XZ); Guangdong Provincial Applied S\&T R\&D Program [grant no. 2016B020237006; to Professor Peng Li (Guangzhou Institutes of Biomedicine and Health, Chinese Academy of Sciences, Guangzhou, China) and XZ].

\section{Availability of data and materials}

The datasets used and/or analyzed during the current study are available from the corresponding author on reasonable request.

\section{Authors' contributions}

YC and XZ designed the study, YC, WY, ZX, WG, DL and $\mathrm{ZL}$ performed the experiments. $\mathrm{YC}$ interpreted the data and drafted the initial manuscript. All authors read and approved the final manuscript.

\section{Ethics approval and consent to participate}

The protocol of the present study was approved by the Ethics Committee of Guangdong Provincial People's Hospital and written informed consent was obtained from all patients.

\section{Patient consent for publication}

Not applicable.

\section{Competing interests}

The authors declare that they have no competing interests.

\section{References}

1. The White House Office of the Press Secretary. Remarks by the President in State of the Union. (2015-01-20) [2015-03-20]. https://www.whitehouse.gov/the-press-office/2015/01/20/remarkspresident-state-union-address-january-20-2015.

2. The White House Office of the Press Secretary. FACT SHEET: President Obama's Precision Medicine Initiative. (2015-01-30) [2015-03-20]. https://www.whitehouse. gov/the-press-office/2015/01/30/fact-sheet-president-obama's-precision-medicine-initiative.

3. Yang Y, Muzny DM, Reid JG, Bainbridge MN, Willis A, Ward PA, Braxton A, Beuten J, Xia F, Niu Z, et al: Clinical whole-exome sequencing for the diagnosis of mendelian disorders. N Engl J Med 369: 1502-1511, 2013.

4. Lee H, Deignan JL, Dorrani N, Strom SP, Kantarci S, Quintero-Rivera F, Das K, Toy T, Harry B, Yourshaw M, et al: Clinical exome sequencing for genetic identification of rare Mendelian disorders. JAMA 312: 1880-1887, 2014.

5. Dewey FE, Grove ME, Pan C, Goldstein BA, Bernstein JA, Chaib H, Merker JD, Goldfeder RL, Enns GM, David SP, et al: Clinical interpretation and implications of whole-genome sequencing. JAMA 311: 1035-1045, 2014.

6. Hayes DN and Kim WY: The next steps in next-gen sequencing of cancer genomes. J Clin Invest 125: 462-468, 2015.

7. Garraway LA. Genomics-driven oncology: Framework for an emerging paradigm. J Clin Oncol 31: 1806-1814, 2013.

8. Good BM, Ainscough BJ, McMichael JF, Su AI and Griffith OL: Organizing knowledge to enable personalization of medicine in cancer. Genome Biol 15: 438, 2014.

9. Griffith M, Miller CA, Griffith OL, Krysiak K, Skidmore ZL, Ramu A, Walker JR, Dang HX, Trani L, Larson DE, et al: Optimizing cancer genome sequencing and analysis. Cell Syst 1: 210-223, 2015.

10. Hyman DM, Solit DB, Arcila ME, Cheng DT, Sabbatini P, Baselga J, Berger MF and Ladanyi M: Precision medicine at memorial sloan kettering cancer center: Clinical next generation sequencing enabling next-generation targeted therapy trials. Drug Discov Today 20: 1422-1428, 2015.

11. Hu Z, Sun R and Curtis C: A population genetics perspective on the determinants of intra-tumor heterogeneity. Biochim Biophys Acta Rev Cancer 1867: 109-126, 2017.

12. Jamal-Hanjani M, Hackshaw A, Ngai Y, Shaw J, Dive C, Quezada S, Middleton G, de Bruin E, Le Quesne J, Shafi S, et al: Tracking genomic cancer evolution for precision medicine: The lung TRACERx Study. PLoS Biol 12: e1001906, 2014.

13. Patel NM, Michelini VV, Snell JM, Balu S, Hoyle AP, Parker JS, Hayward MC, Eberhard DA, Salazar AH, McNeillie P, et al: Enhancing next-generation sequencing-guided cancer care through cognitive computing. Oncologist 23: 179-185, 2018.

14. Pabinger S, Dander A, Fischer M, Snajder R, Sperk M, Efremova M, Krabichler B, Speicher MR, Zschocke J and Trajanoski Z: A survey of tools for variant analysis of next-generation genome sequencing data. Brief Bioinform 15: 256-278, 2014.

15. Oncologists partner with Watson on genomics. Cancer Discov 5: 788,2015

16. Wrzeszczynski KO, Frank MO, Koyama T, Rhrissorrakrai K, Robine N, Utro F, Emde AK, Chen BJ, Arora K, Shah M, et al: Comparing sequencing assays and human-machine analyses in actionable genomics for glioblastoma. Neurol Genet 3: e164, 2017.

17. Itahashi K, Kondo S, Kubo T, Fujiwara Y, Kato M, Ichikawa H, Koyama T, Tokumasu R, Xu J, Huettner CS, et al: Evaluating clinical genome sequence analysis by Watson for genomics. Front Med (Lausanne) 5: 305, 2018. 